A NNALES

UNIVERSITATIS MARIAE CURIE-SKŁODOWSKA

LUBLIN - POLONIA

VOL. LXVIII, NO. 1, 2014

SECTIO A

$31-41$

ANNA MAKAREWICZ, PIOTR PIKUTA and DOMINIK SZAŁKOWSKI

\title{
Properties of the determinant of a rectangular matrix
}

\begin{abstract}
In this paper we present new identities for the Radić's determinant of a rectangular matrix. The results include representations of the determinant of a rectangular matrix as a sum of determinants of square matrices and description how the determinant is affected by operations on columns such as interchanging columns, reversing columns or decomposing a single column.
\end{abstract}

1. Introduction. In [2] Radić introduced the following definition of the determinant of a rectangular matrix.

Definition 1.1. Let $A=\left[A_{1}, A_{2}, \ldots, A_{n}\right]$ be a $m \times n$ matrix with $n$ columns $A_{1}, \ldots, A_{n}$ and $m \leq n$. The determinant of $A$ is defined as

$$
|A|=\sum_{1 \leq j_{1}<\ldots<j_{m} \leq n}(-1)^{r+j_{1}+j_{2}+\ldots+j_{m}}\left|A_{j_{1}}, A_{j_{2}}, \ldots, A_{j_{m}}\right|,
$$

where $r=1+2+\ldots+m$.

The determinant of a square matrix and the determinant (1) of a $m \times n$ matrix, where $m \leq n$, have several common standard properties, including the following (see $[2]$ ):

(1) If a row of $A$ is identical to some other row or is a linear combination of other rows then $|A|=0$.

2000 Mathematics Subject Classification. 15A15.

Key words and phrases. Determinant of rectangular matrix, Radić's determinant. 
(2) If a row of $A$ is multiplied by a number $k$, then the determinant of the resulting matrix is equal to $k|A|$.

(3) Interchanging two rows of $A$ results in changing the sign of the determinant.

(4) The determinant $|A|$ can be calculated using the Laplace expansion.

The properties of the determinant (1) were investigated by Radić $[3,4,5]$ and also by Radić and Sušanj [6]. In the papers cited, the results concerning $2 \times n$ matrices were applied in planar geometry.

Another approach was presented by Amiri, Fathy and Bayat in [1], where the authors proved determinant identities such as Dodgson Condensation Formula and Trahan Formula for rectangular matrices, as well as CauchyBinet Formula for non-square products of two matrices.

In this paper we present new identities for determinants of rectangular matrices. The results include representation of the determinant of a rectangular matrix as a sum of determinants of square matrices and description how the determinant is affected by operations on columns such as interchanging two columns, reversing columns or decomposing a single column.

\section{Properties of the determinant.}

2.1. Representation of the determinant of a rectangular matrix as a sum of determinants of square matrices. For $2 \times n$ matrices, where $n \geq 2$, Radić [3] proved the following theorem.

Theorem 2.1. Let $A=\left[A_{1}, A_{2}, \ldots, A_{n}\right]$ be a $2 \times n$ matrix with $n \geq 2$. Then

$$
\begin{aligned}
|A|= & \left|A_{1}, A_{2}-A_{3}+A_{4}-\ldots+(-1)^{n} A_{n}\right| \\
& +\left|A_{2}, A_{3}-A_{4}+\ldots+(-1)^{n-1} A_{n}\right| \\
& +\ldots \\
& +\left|A_{n-1}, A_{n}\right| .
\end{aligned}
$$

This theorem gives a representation of the determinant of a $2 \times n$ matrix, where $n \geq 2$, as a sum of determinants of square matrices other than the representation (1). We generalize this result to $m \times n$ matrices in the following way.

Theorem 2.2. Let $A=\left[A_{1}, A_{2}, \ldots, A_{n}\right]$ be a $m \times n$ matrix, where $m$ is a number of rows and $n$ is a number of columns, $m \leq n$. Then we have

$$
\begin{aligned}
|A|=\sum_{1 \leq j_{1}<\ldots<j_{m-1}<n}(-1)^{r+j_{1}+j_{2}+\ldots+j_{m-1}} & \\
& \times\left|A_{j_{1}}, A_{j_{2}}, \ldots, A_{j_{m-1}}, \sum_{k=j_{m-1}+1}^{n}(-1)^{k} A_{k}\right| .
\end{aligned}
$$


Proof. Applying (1), we have

$$
\begin{aligned}
|A| & =\sum_{1 \leq j_{1}<\ldots<j_{m} \leq n}(-1)^{r+j_{1}+j_{2}+\ldots+j_{m}}\left|A_{j_{1}}, A_{j_{2}}, \ldots, A_{j_{m}}\right| \\
& =\sum_{1 \leq j_{1}<j_{2}<\ldots<j_{m-1}<n} \sum_{k=j_{m-1}+1}^{n}(-1)^{r+j_{1}+j_{2}+\ldots+j_{m-1}+k} \\
& \quad \times\left|A_{j_{1}}, A_{j_{2}}, \ldots, A_{j_{m-1}}, A_{k}\right| \\
& \quad \sum_{1 \leq j_{1}<\ldots<j_{m-1}<n}(-1)^{r+j_{1}+j_{2}+\ldots+j_{m-1}} \\
& \times\left|A_{j_{1}}, A_{j_{2}}, \ldots, A_{j_{m-1}}, \sum_{k=j_{m-1}+1}^{n}(-1)^{k} A_{k}\right| .
\end{aligned}
$$

Using the same method, one can easily prove the following two theorems.

Theorem 2.3. Let $A=\left[A_{1}, A_{2}, \ldots, A_{n}\right]$ be a $m \times n$ matrix, $m \leq n$. Then we have

$$
|A|=\sum_{1<j_{2}<\ldots<j_{m} \leq n}(-1)^{r+j_{2}+j_{3}+\ldots+j_{m}}\left|\sum_{k=1}^{j_{2}-1}(-1)^{k} A_{k}, A_{j_{2}}, \ldots, A_{j_{m}}\right|,
$$

where $r=1+2+\ldots+m$.

Theorem 2.4. Let $A=\left[A_{1}, A_{2}, \ldots, A_{n}\right]$ be a $m \times n$ matrix, $m \leq n$. Then for each $p \in\{2,3, \ldots, m-1\}$ we have

$$
\begin{aligned}
& |A|=\sum_{\substack{1 \leq j_{1}<\ldots<j_{p-1} \\
j_{p+1}<\ldots<j_{m} \leq n \\
j_{p+1}-j_{p-1}>1}}(-1)^{r+j_{1}+j_{2}+\ldots+j_{p-1}+j_{p+1}+\ldots+j_{m}} \\
& \quad \times\left|A_{j_{1}}, \ldots, A_{j_{p-1}}, \sum_{k=j_{p-1}+1}^{j_{p+1}-1}(-1)^{k} A_{k}, A_{j_{p+1}}, \ldots, A_{j_{m}}\right|,
\end{aligned}
$$

where $r=1+2+\ldots+m$.

Example 1. Let $\left[A_{1}, A_{2}, A_{3}, A_{4}\right]$ be a $3 \times 4$ matrix. Then

$$
\begin{aligned}
\left|A_{1}, A_{2}, A_{3}, A_{4}\right| & =\left|A_{1}, A_{2}, A_{3}-A_{4}\right|+\left|A_{1}, A_{3}, A_{4}\right|-\left|A_{2}, A_{3}, A_{4}\right| \\
& =\left|A_{1}, A_{2}, A_{3}\right|-\left|A_{1}, A_{2}, A_{4}\right|+\left|A_{1}-A_{2}, A_{3}, A_{4}\right| \\
& =\left|A_{1}, A_{2}, A_{3}\right|-\left|A_{1}, A_{2}-A_{3}, A_{4}\right|-\left|A_{2}, A_{3}, A_{4}\right| .
\end{aligned}
$$

2.2. Decomposing a column. If a column $K$ in a square matrix $A$ is a sum of two columns (eg. $K=K_{1}+K_{2}$ ), then the determinant $|A|$ is a sum of two determinants of matrices obtained from $A$ by replacing $K$ by $K_{1}$ and $K_{2}$ respectively.

For rectangular matrices we have a similar property. 
Theorem 2.5. Let $A=\left[A_{1}, A_{2}, \ldots, A_{k}, \ldots, A_{n}\right]$ be a $m \times n$ matrix, $m \leq n$, and $A_{k}=B_{k}+C_{k}$ for some $k \in\{1,2, \ldots, n\}$. Then

$$
\begin{aligned}
|A|= & \left|A_{1}, A_{2}, \ldots, A_{k-1}, B_{k}, A_{k+1}, \ldots, A_{n}\right| \\
& +\left|A_{1}, A_{2}, \ldots, A_{k-1}, C_{k}, A_{k+1}, \ldots, A_{n}\right| \\
& +\sum_{\substack{1 \leq j_{1}<\ldots<j_{m} \leq n \\
k \notin\left\{j_{1} \ldots ., j_{m}\right\}}}(-1)^{r+j_{1}+j_{2}+\ldots+j_{m}+1}\left|A_{j_{1}}, A_{j_{2}}, \ldots, A_{j_{m}}\right|,
\end{aligned}
$$

where $r=1+2+\ldots+m$.

Proof. After applying (1)

$$
|A|=\sum_{1 \leq j_{1}<\ldots<j_{m} \leq n}(-1)^{r+j_{1}+j_{2}+\ldots+j_{m}}\left|A_{j_{1}}, A_{j_{2}}, \ldots, A_{j_{m}}\right|
$$

we separate the sum of determinants into two sums: the first one consisting of the determinants of matrices which contain the column $A_{k}=B_{k}+C_{k}$ and the second one consisting of other determinants.

$$
\begin{aligned}
|A|= & \sum_{\substack{1 \leq j_{1}<\ldots<j_{m} \leq n \\
k \in\left\{j_{1}, \ldots, j_{m}\right\}}}(-1)^{r+j_{1}+j_{2}+\ldots+j_{m}}\left|A_{j_{1}}, \ldots, A_{k}, \ldots, A_{j_{m}}\right| \\
& +\sum_{\substack{1 \leq j_{1}<\ldots<j_{m} \leq n \\
k \notin\left\{j_{1}, \ldots, j_{m}\right\}}}(-1)^{r+j_{1}+j_{2}+\ldots+j_{m}}\left|A_{j_{1}}, A_{j_{2}}, \ldots, A_{j_{m}}\right| \\
= & \sum_{\substack{1 \leq j_{1}<\ldots<j_{m} \leq n \\
k \in\left\{j_{1}, \ldots, j_{m}\right\}}}(-1)^{r+j_{1}+j_{2}+\ldots+j_{m}}\left|A_{j_{1}}, \ldots, B_{k}, \ldots, A_{j_{m}}\right| \\
& +\sum_{\substack{1 \leq j_{1}<\ldots<j_{m} \leq n \\
k \in\left\{j_{1}, \ldots, j_{m}\right\}}}(-1)^{r+j_{1}+j_{2}+\ldots+j_{m}}\left|A_{j_{1}}, \ldots, C_{k}, \ldots, A_{j_{m}}\right| \\
& +\sum_{\substack{1 \leq j_{1}<\ldots<j_{m} \leq n \\
k \notin\left\{j_{1}, \ldots, j_{m}\right\}}}(-1)^{r+j_{1}+j_{2}+\ldots+j_{m}}\left|A_{j_{1}}, A_{j_{2}}, \ldots, A_{j_{m}}\right| .
\end{aligned}
$$

Now the third sum is added and subtracted so that it can be included into both the first and the second sum:

$$
\begin{aligned}
|A|=\mid & A_{1}, A_{2}, \ldots, A_{k-1}, B_{k}, A_{k+1}, \ldots, A_{n} \mid \\
& +\left|A_{1}, A_{2}, \ldots, A_{k-1}, C_{k}, A_{k+1}, \ldots, A_{n}\right| \\
& -\sum_{\substack{1 \leq j_{1}<\ldots<j_{m} \leq n \\
k \notin\left\{j_{1}, \ldots, j_{m}\right\}}}(-1)^{r+j_{1}+j_{2}+\ldots+j_{m}}\left|A_{j_{1}}, A_{j_{2}}, \ldots, A_{j_{m}}\right|
\end{aligned}
$$




$$
\begin{aligned}
= & \left|A_{1}, A_{2}, \ldots, A_{k-1}, B_{k}, A_{k+1}, \ldots, A_{n}\right| \\
& +\left|A_{1}, A_{2}, \ldots, A_{k-1}, C_{k}, A_{k+1}, \ldots, A_{n}\right| \\
& +\sum_{\substack{1 \leq j_{1}<\ldots<j_{m} \leq n \\
k \notin\left\{j_{1}, \ldots, j_{m}\right\}}}(-1)^{r+j_{1}+j_{2}+\ldots+j_{m}+1}\left|A_{j_{1}}, A_{j_{2}}, \ldots, A_{j_{m}}\right| .
\end{aligned}
$$

Example 2. Let $\left[A_{1}, A_{2}, A_{3}\right]$ be a $2 \times 3$ matrix and $A_{1}=B_{1}+C_{1}$. Then according to Theorem 2.5 we have

$$
\begin{aligned}
\left|B_{1}+C_{1}, A_{2}, A_{3}\right|= & \left|B_{1}, A_{2}, A_{3}\right|+\left|C_{1}, A_{2}, A_{3}\right| \\
& +\sum_{\substack{1 \leq j_{1}<j_{2} \leq 3 \\
1 \notin\left\{j_{1}, j_{2}\right\}}}(-1)^{(1+2)+j_{1}+j_{2}+1}\left|A_{j_{1}}, A_{j_{2}}\right| \\
= & \left|B_{1}, A_{2}, A_{3}\right|+\left|C_{1}, A_{2}, A_{3}\right|+(-1)^{3+2+3+1}\left|A_{2}, A_{3}\right| \\
= & \left|B_{1}, A_{2}, A_{3}\right|+\left|C_{1}, A_{2}, A_{3}\right|-\left|A_{2}, A_{3}\right| .
\end{aligned}
$$

2.3. Interchanging columns. Interchanging columns in a square matrix results in changing the sign of the determinant. Rectangular matrices in which the number of columns is equal to the number of rows increased by one have the same property.

Theorem 2.6. Let $A=\left[A_{1}, A_{2}, \ldots, A_{m}, A_{m+1}\right]$ be a $m \times(m+1)$ matrix. Then for each $i, j \in\{1,2, \ldots, m+1\}$ such that $i<j$, we have

$$
|A|=-\left|A_{1}, A_{2}, \ldots, A_{i-1}, A_{j}, A_{i+1}, \ldots, A_{j-1}, A_{i}, A_{j+1}, \ldots, A_{m}, A_{m+1}\right| .
$$

Proof. Let $r=1+2+\ldots+m$. Fix $i, j \in\{1,2, \ldots, m+1\}$ such that $i<j$. From all the determinants in the right-hand side of

$$
|A|=\sum_{1 \leq j_{1}<\ldots<j_{m} \leq n}(-1)^{r+j_{1}+j_{2}+\ldots+j_{m}}\left|A_{j_{1}}, A_{j_{2}}, \ldots, A_{j_{m}}\right|,
$$

we distinguish determinants of two matrices which contain either $A_{i}$ or $A_{j}$ but not both of them. Thus we have

$$
\begin{aligned}
|A|=(-1)^{\left[r+\frac{(m+1)(m+2)}{2}-i\right]} & \\
& \times\left|A_{1}, A_{2}, \ldots, A_{i-1}, A_{i+1}, \ldots, A_{j-1}, A_{j}, A_{j+1}, \ldots, A_{m+1}\right| \\
& +(-1)^{\left[r+\frac{(m+1)(m+2)}{2}-j\right]} \\
& \times\left|A_{1}, A_{2}, \ldots, A_{i-1}, A_{i}, A_{i+1}, \ldots, A_{j-1}, A_{j+1}, \ldots, A_{m+1}\right| \\
& +\sum_{\substack{1 \leq j_{1}<\ldots<j_{m} \leq n \\
i, j \in\left\{j_{1}, \ldots, j_{m}\right\}}}(-1)^{r+j_{1}+j_{2}+\ldots+j_{m}}\left|A_{j_{1}}, \ldots, A_{i}, \ldots, A_{j}, \ldots A_{j_{m}}\right| .
\end{aligned}
$$


Notice that exactly $j-i-1$ inversions are needed to move the column $A_{j}$ to the position between $A_{i-1}$ and $A_{i+1}$ in the first summand. Similarly, in the second summand, also $j-i-1$ inversions are needed to move the column $A_{i}$ to the position between $A_{j-1}$ and $A_{j+1}$.

In other summands we can simply interchange columns $A_{i}$ and $A_{j}$ with the sign change. Thus we have

$$
\begin{aligned}
& |A|=(-1)^{\left[r+\frac{(m+1)(m+2)}{2}-i+(j-i+1)\right]} \\
& \times\left|A_{1}, A_{2}, \ldots, A_{i-1}, A_{j}, A_{i+1}, \ldots, A_{j-1}, A_{j+1}, \ldots, A_{m+1}\right| \\
& +(-1)^{\left[r+\frac{(m+1)(m+2)}{2}-j+(j-i+1)\right]} \\
& \times\left|A_{1}, A_{2}, \ldots, A_{i-1}, A_{i+1}, \ldots, A_{j-1}, A_{i}, A_{j+1}, \ldots, A_{m+1}\right| \\
& -\sum_{\substack{1 \leq j_{1}<\ldots<j_{m} \leq n \\
i, j \in\left\{j_{1}, \ldots, j_{m}\right\}}}(-1)^{r+j_{1}+j_{2}+\ldots+j_{m}}\left|A_{j_{1}}, \ldots, A_{j}, \ldots, A_{i}, \ldots A_{j_{m}}\right| \\
& =-(-1)^{\left[r+\frac{(m+1)(m+2)}{2}-j\right]} \\
& \times\left|A_{1}, A_{2}, \ldots, A_{i-1}, A_{j}, A_{i+1}, \ldots, A_{j-1}, A_{j+1}, \ldots, A_{m+1}\right| \\
& -(-1)^{\left[r+\frac{(m+1)(m+2)}{2}-i\right]} \\
& \times\left|A_{1}, A_{2}, \ldots, A_{i-1}, A_{i+1}, \ldots, A_{j-1}, A_{i}, A_{j+1}, \ldots, A_{m+1}\right| \\
& -\sum_{\substack{1 \leq j_{1}<\ldots<j_{m} \leq n \\
i, j \in\left\{j_{1}, \ldots, j_{m}\right\}}}(-1)^{r+j_{1}+j_{2}+\ldots+j_{m}}\left|A_{j_{1}}, \ldots, A_{j}, \ldots, A_{i}, \ldots A_{j_{m}}\right| \\
& =-\left|A_{1}, A_{2}, \ldots, A_{i-1}, A_{j}, A_{i+1}, \ldots, A_{j-1}, A_{i}, A_{j+1}, \ldots, A_{m}, A_{m+1}\right| .
\end{aligned}
$$

Consider a $m \times n$ matrix $A$ with $m$ rows and $n$ columns, $m \leq n$. Let $A^{\prime}$ be a matrix obtained from $A$ by interchanging two columns. Theorem 2.6 tells us that $|A|+\left|A^{\prime}\right|=0$ when $n-m=1$. However, in general, if $n-m>1$ the sum $|A|+\left|A^{\prime}\right|$ is not zero.

For a $m \times n$ matrix $M=\left[M_{1}, M_{2}, \ldots, M_{n}\right]$ and each $i, j \in\{1,2, \ldots, m\}$, such that $i<j$, denote

$$
S_{1}(M, i, j)=\sum_{\substack{1 \leq j_{1}<\ldots<j_{m} \leq n \\ i, j \notin\left\{j_{1}, \ldots, j_{m}\right\}}}(-1)^{r+j_{1}+j_{2}+\ldots+j_{m}}\left|M_{j_{1}}, M_{j_{2}}, \ldots, M_{j_{m}}\right|,
$$




$$
\begin{aligned}
S_{2}(M, i, j)= & \sum_{\substack{1 \leq j_{1}<\ldots<j_{m} \leq n \\
i, j \in\left\{j_{1}, \ldots, j_{m}\right\}}}(-1)^{r+j_{1}+j_{2}+\ldots+j_{m}}\left|M_{j_{1}}, M_{j_{2}}, \ldots, M_{j_{m}}\right|, \\
S_{3}(M, i, j)= & \sum_{\substack{1 \leq j_{1}<\ldots<j_{m} \leq n \\
(i \in J, j \notin J \text { or } i \notin J, j \in J) \\
J=\{i, \ldots, j\} \backslash\left\{j_{1}, \ldots, j_{m}\right\} \\
\operatorname{card}(J) \equiv 1(\bmod 2)}}(-1)^{r+j_{1}+j_{2}+\ldots+j_{m}}\left|M_{j_{1}}, M_{j_{2}}, \ldots, M_{j_{m}}\right|, \\
S_{4}(M, i, j)= & \sum_{\substack{1 \leq j_{1}<\ldots<j_{m} \leq n \\
(i \in J, j \notin J \operatorname{or} i \notin J, j \in J) \\
J=\{i, \ldots, j\} \backslash\left\{j_{1}, \ldots, j_{m}\right\} \\
\operatorname{card}(J) \equiv 0(\bmod 2)}}(-1)^{r+j_{1}+j_{2}+\ldots+j_{m}}\left|M_{j_{1}}, M_{j_{2}}, \ldots, M_{j_{m}}\right|,
\end{aligned}
$$

where $r=1+2+\ldots+m$ and $\operatorname{card}(X)$ stands for the cardinality of $X$.

Theorem 2.7. Let $A=\left[A_{1}, A_{2}, \ldots, A_{n}\right]$ be a $m \times n$ matrix with $m$ rows and $n$ columns, $m \leq n$. For $i, j \in\{1,2, \ldots, n\}$ such that $i \neq j$ denote by $A_{A_{i} \leftrightarrow A_{j}}$ the matrix obtained from $A$ by interchanging columns $A_{i}$ and $A_{j}$. Then

$$
\begin{aligned}
|A|+\left|A_{A_{i} \leftrightarrow A_{j}}\right| & =2 S_{1}(A, i, j)+2 S_{4}(A, i, j) \\
& =2 S_{1}\left(A_{A_{i} \leftrightarrow A_{j}}, i, j\right)+2 S_{4}\left(A_{A_{i} \leftrightarrow A_{j}}, i, j\right) .
\end{aligned}
$$

Proof. Fix $i, j \in\{1,2, \ldots, m\}$ such that $i<j$. (If $i>j$ we can proceed analogously). We have

$$
|A|=S_{1}(A, i, j)+S_{2}(A, i, j)+S_{3}(A, i, j)+S_{4}(A, i, j) .
$$

It is easy to verify that

$$
\begin{aligned}
& S_{1}\left(A_{A_{i} \leftrightarrow A_{j}}, i, j\right)=S_{1}(A, i, j), \\
& S_{2}\left(A_{A_{i} \leftrightarrow A_{j}}, i, j\right)=-S_{2}(A, i, j) .
\end{aligned}
$$

Notice that each of the matrices in $S_{3}(A, i, j)+S_{4}(A, i, j)$ needs exactly $(j-i-\operatorname{card}(J))$ column inversions to move the column $A_{i}$ to the position where $A_{j}$ would be, and also $(j-i-\operatorname{card}(J))$ inversions are needed to move the column $A_{j}$ to the position where $A_{i}$ would be. 
Therefore,

$$
\begin{aligned}
& S_{3}(A, i, j)=\sum_{1 \leq j_{1}<\ldots<j_{m} \leq n}(-1)^{r+\left(\sum_{k=1}^{m} j_{k}+j\right)-j+(j-i-\operatorname{card}(J))} \\
& J=\{i, \ldots, j\} \backslash\left\{j_{1}, \ldots, j_{m}\right\} \\
& \operatorname{card}(J) \equiv 1(\bmod 2) \quad \times\left|A_{j_{1}}, \ldots, A_{j_{p}}, A_{i}, A_{j_{q}}, \ldots, A_{j_{m}}\right| \\
& +\sum_{\substack{1 \leq j_{1}<\ldots<j_{m} \leq n \\
i \notin J, j \in J}}(-1)^{r+\left(\sum_{k=1}^{m} j_{k}+i\right)-i+(j-i-\operatorname{card}(J))} \\
& J=\{i, \ldots, j\} \backslash\left\{j_{1}, \ldots, j_{m}\right\} \\
& \operatorname{card}(J) \equiv 1(\bmod 2) \quad \times\left|A_{j_{1}}, \ldots, A_{j_{u}}, A_{j}, A_{j_{v}}, \ldots, A_{j_{m}}\right| \\
& =-\sum_{\substack{1 \leq j_{1}<\ldots<j_{m} \leq n \\
i \in J, j \notin J}}(-1)^{r+\left(\sum_{k=1}^{m} j_{k}+j\right)-i} \\
& J=\{i, \ldots, j\} \backslash\left\{j_{1}, \ldots, j_{m}\right\} \\
& \operatorname{card}(J) \equiv 1(\bmod 2) \quad \times\left|A_{j_{1}}, \ldots, A_{j_{p}}, A_{i}, A_{j_{q}}, \ldots, A_{j_{m}}\right| \\
& -\sum_{\substack{1 \leq j_{1}<\ldots<j_{m} \leq n \\
i \notin J, j \in J}}(-1)^{r+\left(\sum_{k=1}^{m} j_{k}+i\right)-j} \\
& J=\{i, \ldots, j\} \backslash\left\{j_{1}, \ldots, j_{m}\right\} \\
& \operatorname{card}(J) \equiv 1(\bmod 2) \\
& \times\left|A_{j_{1}}, \ldots, A_{j_{u}}, A_{j}, A_{j_{v}}, \ldots, A_{j_{m}}\right| \\
& =-S_{3}\left(A_{A_{i} \leftrightarrow A_{j}}, i, j\right),
\end{aligned}
$$

where $r=1+2+\ldots+m$ and $j_{p}<j<j_{q}, j_{u}<i<j_{v}$ for some $p, q, u, v$.

Similarly, we have

and finally,

$$
S_{4}\left(A_{A_{i} \leftrightarrow A_{j}}, i, j\right)=S_{4}(A, i, j)
$$

$$
\begin{aligned}
|A|+\left|A_{A_{i} \leftrightarrow A_{j}}\right|= & S_{1}(A, i, j)+S_{2}(A, i, j)+S_{3}(A, i, j)+S_{4}(A, i, j) \\
& +S_{1}\left(A_{A_{i} \leftrightarrow A_{j}}, i, j\right)+S_{2}\left(A_{A_{i} \leftrightarrow A_{j}}, i, j\right) \\
& +S_{3}\left(A_{A_{i} \leftrightarrow A_{j}}, i, j\right)+S_{4}\left(A_{A_{i} \leftrightarrow A_{j}}, i, j\right) \\
= & 2 S_{1}(A, i, j)+2 S_{4}(A, i, j) .
\end{aligned}
$$


Corollary 2.8. Let $A$ be a $m \times n$ matrix, $m \leq n$. If $i, j \in\{1,2, \ldots, n\}$ satisfy $|i-j|=1$, then

$$
|A|+\left|A_{A_{i} \leftrightarrow A_{j}}\right|=2 S_{1}(A, i, j)=2 S_{1}\left(A_{A_{i} \leftrightarrow A_{j}}, i, j\right) .
$$

Example 3. Below we present a few identities obtained from Theorem 2.6, Theorem 2.7 and Corollary 2.8.

(a) Let $\left[A_{1}, A_{2}, A_{3}, A_{4}, A_{5}\right]$ be a $4 \times 5$ matrix. Then

$$
\left|A_{1}, A_{2}, A_{3}, A_{4}, A_{5}\right|=-\left|A_{5}, A_{2}, A_{3}, A_{4}, A_{1}\right|=\left|A_{5}, A_{4}, A_{3}, A_{2}, A_{1}\right| \text {. }
$$

(b) Let $\left[A_{1}, A_{2}, A_{3}, A_{4}\right]$ be a $2 \times 4$ matrix. Then

$$
\begin{aligned}
\left|A_{1}, A_{2}, A_{3}, A_{4}\right|+\left|A_{2}, A_{1}, A_{3}, A_{4}\right| & =2\left|A_{3}, A_{4}\right|, \\
\left|A_{1}, A_{2}, A_{3}, A_{4}\right|+\left|A_{1}, A_{4}, A_{3}, A_{2}\right|= & 2\left(\left|A_{1}, A_{2}\right|-\left|A_{1}, A_{3}\right|+\left|A_{1}, A_{4}\right|\right), \\
\left|A_{1}, A_{2}, A_{3}, A_{4}\right|+\left|A_{4}, A_{2}, A_{3}, A_{1}\right|= & 2\left(\left|A_{1}, A_{2}\right|-\left|A_{1}, A_{3}\right|+\left|A_{2}, A_{3}\right|\right. \\
& \left.-\left|A_{2}, A_{4}\right|+\left|A_{3}, A_{4}\right|\right) .
\end{aligned}
$$

2.4. Reversing columns. Reversing columns in a $n \times n$ square matrix results in changing the sign of its determinant if and only if $n$ is congruent to 2 or $3(\bmod 4)$. Surprisingly, the determinant of a rectangular matrix also either changes or does not change the sign after column reversing, depending on the number of rows and the number of columns of the matrix.

Theorem 2.9. Let $\left[A_{1}, A_{2}, \ldots, A_{n}\right]$ be a $m \times n$ matrix, $m \leq n$. Then we have

$$
\begin{aligned}
\left|A_{n}, A_{n-1}, \ldots, A_{2}, A_{1}\right|=\left|A_{1}, A_{2}, \ldots, A_{n-1}, A_{n}\right| \cdot(-1)^{\frac{m}{2}(2 n+m+1)} \\
= \begin{cases}\left|A_{1}, A_{2}, \ldots, A_{n-1}, A_{n}\right| & \text { if } m \equiv 0(\bmod 4), \\
\left|A_{1}, A_{2}, \ldots, A_{n-1}, A_{n}\right| \cdot(-1)^{n+1} & \text { if } m \equiv 1(\bmod 4), \\
\left|A_{1}, A_{2}, \ldots, A_{n-1}, A_{n}\right| \cdot(-1) & \text { if } m \equiv 2(\bmod 4), \\
\left|A_{1}, A_{2}, \ldots, A_{n-1}, A_{n}\right| \cdot(-1)^{n} & \text { if } m \equiv 3(\bmod 4) .\end{cases}
\end{aligned}
$$

Proof. Let $r=1+2+\ldots+m=\frac{m(m+1)}{2}$ and $B_{k}=A_{n+1-k}, k \in\{1,2, \ldots, n\}$. Since exactly $(m-1)+(m-2)+\ldots+1=\frac{(m-1) m}{2}$ inversions of (adjacent) columns are needed to reverse the columns of a $m \times m$ matrix, we have

$$
\begin{aligned}
\left|B_{1}, B_{2}, \ldots, B_{n}\right| & =\sum_{1 \leq i_{1}<\ldots<i_{m} \leq n}(-1)^{r+i_{1}+i_{2}+\ldots+i_{m}}\left|B_{i_{1}}, B_{i_{2}}, \ldots, B_{i_{m}}\right| \\
& =\sum_{1 \leq i_{1}<\ldots<i_{m} \leq n}(-1)^{r+i_{1}+i_{2}+\ldots+i_{m}+\frac{(m-1) m}{2}} \\
& \times \sum_{1 \leq i_{1}<\ldots<i_{m} \leq n}(-1)^{r+i_{1}+i_{2}+\ldots+i_{m}+\frac{(m-1) m}{2}} \\
& \times\left|A_{i_{m-1}}, \ldots, B_{i_{1}}\right| \\
&
\end{aligned}
$$


Applying the following change of variables: $j_{k}=n+1-i_{m-k+1}$ for each $k \in\{1,2, \ldots, m\}$, we get

$$
\begin{aligned}
\mid A_{n}, & A_{n-1}, \ldots, A_{2}, A_{1}|=| B_{1}, B_{2}, \ldots, B_{n} \mid \\
& =\sum_{1 \leq j_{1}<\ldots<j_{m} \leq n}(-1)^{r+m(n+1)-\left(j_{1}+j_{2}+\ldots+j_{m}\right)+\frac{(m-1) m}{2}}\left|A_{j_{1}}, A_{j_{2}}, \ldots, A_{j_{m}}\right| \\
& =\left|A_{1}, A_{2}, \ldots, A_{n-1}, A_{n}\right| \cdot(-1)^{m(n+1)+\frac{(m-1) m}{2}} \\
& =\left|A_{1}, A_{2}, \ldots, A_{n-1}, A_{n}\right| \cdot(-1)^{\frac{m}{2}(2 n+m+1)} .
\end{aligned}
$$

Finally, we state that

$$
(-1)^{\frac{m}{2}(2 n+m+1)}= \begin{cases}1 & \text { if } m \equiv 0(\bmod 4) \\ (-1)^{n+1} & \text { if } m \equiv 1(\bmod 4) \\ (-1) & \text { if } m \equiv 2(\bmod 4) \\ (-1)^{n} & \text { if } m \equiv 3(\bmod 4)\end{cases}
$$

which is easy to verify.

Example 4. Let

$$
\left[A_{1}, A_{2}, A_{3}, A_{4}, A_{5}, A_{6}, A_{7}, A_{8}, A_{9}\right]
$$

be a $5 \times 9$ matrix. Then

$$
\left|A_{1}, A_{2}, A_{3}, A_{4}, A_{5}, A_{6}, A_{7}, A_{8}, A_{9}\right|=\left|A_{9}, A_{8}, A_{7}, A_{6}, A_{5}, A_{4}, A_{3}, A_{2}, A_{1}\right| .
$$

\section{REFERENCES}

[1] Amiri, M., Fathy, M., Bayat, M., Generalization of some determinantal identities for non-square matrices based on Radic's definition, TWMS J. Pure Appl. Math. 1, no. 2 (2010), 163-175.

[2] Radić, M., A definition of determinant of rectangular matrix, Glas. Mat. Ser. III 1(21) (1966), 17-22.

[3] Radić, M., About a determinant of rectangular $2 \times n$ matrix and its geometric interpretation, Beiträge Algebra Geom. 46, no. 2 (2005), 321-349.

[4] Radić, M., Areas of certain polygons in connection with determinants of rectangular matrices, Beiträge Algebra Geom. 49, no. 1 (2008), 71-96.

[5] Radić, M., Certain equalities and inequalities concerning polygons in $\mathbb{R}^{2}$, Beiträge Algebra Geom. 50, no. 1 (2009), 235-248.

[6] Radić, M., Sušanj, R., Geometrical meaning of one generalization of the determinant of a square matrix, Glas. Mat. Ser. III 29(49), no. 2 (1994), 217-233. 
Anna Makarewicz

Lublin University of Technology

Department of Applied Mathematics

ul. Nadbystrzycka $38 \mathrm{D}$

20-618 Lublin

Poland

e-mail: anna_makarewicz@o2.p1

Piotr Pikuta

Institute of Mathematics

Maria Curie-Skłodowska University

pl. Marii Curie-Skłodowskiej 1

20-031 Lublin

Poland

e-mail: ppikuta@poczta.umcs.lublin.pl

Dominik Szałkowski

Institute of Mathematics

Maria Curie-Skłodowska University

pl. Marii Curie-Skłodowskiej 1

20-031 Lublin

Poland

e-mail: dominik.szalkowski@umcs.lublin.pl

Received February 21, 2013 